\title{
Severe fever with thrombocytopenia syndrome: comparison with scrub typhus and clinical diagnostic prediction
}

Sang-Won Park' ${ }^{1,2}$, Chang-Seop Lee ${ }^{3}$, Jeong-Han Kim', In-Gyu Bae ${ }^{4}$, Chisook Moon ${ }^{5}$, Yee Gyung Kwak ${ }^{5}$, Baek-Nam Kim ${ }^{5}$, Jae Hoon Lee ${ }^{6}$, Seong Yeol Ryu ${ }^{7}$, Hee-Chang Jang ${ }^{8}$, Jian Hur ${ }^{9}$, Jae-Bum Jun ${ }^{10}$, Younghee Jung ${ }^{11}$, Hyun-Ha Chang ${ }^{12}$, Young Keun Kim ${ }^{13}$, Jeong-Hwan Hwang ${ }^{3}$, Yeon-Sook Kim ${ }^{14}$, Hye Won Jeong ${ }^{15}$,

Kyoung-Ho Song ${ }^{16}$, Wan Beom Park ${ }^{1}$, Eu Suk Kim ${ }^{16}$ and Myoung-don Oh ${ }^{1^{*}}$ (D)

\begin{abstract}
Background: Severe fever with thrombocytopenia syndrome (SFTS) is emerging in Asian 3 countries, China, Japan and Korea, which are scrub typhus endemic areas, and its incidence is increasing. As the two infections overlap epidemiologically and clinically and the accessibility or sensitivity of diagnostic tests is limited, early clinical prediction may be useful for diagnostic and therapeutic purposes.

Methods: Patients aged $\geq 16$ years who were clinically suspected and laboratory-confirmed to be infected with Orientia tsutsugamushi or the SFTS virus in South Korea were enrolled. Clinical and laboratory parameters were compared. Scrub typhus was further subclassified according to the status of eschar and skin rash. An SFTS prediction scoring tool was generated based on a logistic regression analysis of SFTS compared with scrub typhus.

Results: The analysis was performed on 255 patients with scrub typhus and 107 patients with SFTS. At initial presentation, subjective symptoms except for gastrointestinal symptoms, were more prominent in scrub typhus patients. In addition to the characteristic eschar and skin rash, headache was significantly more prominent in scrub typhus, while laboratory abnormalities were more prominent in SFTS. Leukopenia (white blood cell count $<4000 / \mathrm{mm}^{3}$; odds ratio [OR] 30.13), thrombocytopenia (platelet count $<80,000 / \mathrm{mm}^{3}$; OR 19.73) and low C-reactive protein $(<1 \mathrm{mg} / \mathrm{dL}$; OR 67.46) were consistent risk factors for SFTS (all $P<0.001)$. A prediction score was generated using these 3 variables, and a score $\geq 2$ had a sensitivity of $93.1 \%$ (95\% confidence interval [CI], 87.9-96.4\%) and a specificity of $96.1 \%(95 \% \mathrm{Cl}, 93.8-97.6 \%)$ for SFTS.
\end{abstract}

Conclusion: This prediction scoring tool may be useful for differentiating SFTS from eschar- or skin rash-negative scrub typhus. It is a simple and readily applicable tool with potential for use in primary care settings.

Keywords: SFTS, Severe fever with thrombocytopenia syndrome, Scrub typhus, Tsutsugamushi, Korea, Prediction, Score

\section{Background}

Severe fever with thrombocytopenia syndrome (SFTS) is an emerging infectious disease that is caused by the SFTS virus (SFTSV); it is endemic in 3 East Asian countries: China, Korea and Japan [1-3]. The incidence of SFTS is increasing, and the case-fatality rate ranges from 5.3 to $32.6 \%$ [4-6]; however, there are not yet effective antiviral

\footnotetext{
* Correspondence: mdohmd@snu.ac.kr

${ }^{1}$ Department of Internal Medicine, Seoul National University College of Medicine, 103 Daehak-ro, Jongno-gu, Seoul 03080, the Republic of Korea Full list of author information is available at the end of the article
}

therapeutics or a vaccine [7]. SFTS was listed as a priority disease that requires urgent research and development by the World Health Organization in 2017 [8]. Orientia tsutsugamushi is endemic to these 3 countries, which is a leading cause of treatable non-malarial febrile illness in Asia [9]. In 2017, 10,528 cases of scrub typhus were reported in South Korea. Eschar and a maculopapular skin rash are characteristic findings of this disease and are critical clues for its diagnosis. The case-fatality rate of scrub typhus has a median of $6.0 \%$ in untreated cases and $1.4 \%$ in treated cases [9]. 
Although SFTSV and O. tsutsugamushi do not share specific vectors, they are transmitted to humans through ticks and mites bites mostly, respectively, during outdoor activities. The ecological differences between vectors may characterize their epidemiological features, including the region of infection and peak epidemic seasons. However, there are considerable overlaps of their epidemiological and clinical features, which makes their differential diagnosis difficult, particularly during the high epidemic season of scrub typhus. Patients with SFTS have the potential to deteriorate during the second week of the illness [5], and early diagnosis of SFTS may lead to early investigational therapeutics and stricter infection control measures to prevent human-to-human transmission [10-13]. However, the sensitivity of diagnostic assays for scrub typhus is low [14]. The confirmatory test for SFTS is usually performed in the national reference laboratory, and a serologic assay for the point of care is not yet commercially available. Therefore, only a high index of clinical suspicion may lead to a rapid clinical decision or an early referral, particularly in primary care settings.

Clinical diagnostic prediction based on the features differentiating SFTS from scrub typhus in endemic areas, particularly during the overlap period, may be clinically useful to guide the diagnostic and therapeutic strategies in the absence of rapid point-of-care diagnostic test. This study compared the clinical and laboratory features of the two diseases and constructed a clinical prediction tool composed of a scoring system for SFTS. We performed several subgroup analyses, including for eschar-negative scrub typhus, which is difficult to suspect clinically because it lacks critical clues.

\section{Methods}

\section{Patients}

Patients in South Korea aged $\geq 16$ years who were clinically suspected and laboratory-confirmed to be infected with O. tsutsugamushi or SFTSV were enrolled. Cases of eschar-positive and -negative scrub typhus were prospectively included from 8 community-based hospitals in 2006; part of this study was previously published [15]. Additional patients with only eschar-negative scrub typhus were prospectively included from 6 communitybased hospitals from 2009 to 2011; these patients had been thoroughly examined and cared for by the infectious diseases specialists in charge. The participating hospitals in both studies were Chonbuk National University Hospital, Dankook University Hospital, Dongguk University Ilsan Hospital, Ilsan Paik Hospital, Namwon Medical Center, Pusan Paik Hospital, Sanggye Paik Hospital, Sunlin Hospital, Boramae Medical Center, and Wonkwang University Hospital. SFTS cases were retrospectively collected from 36 hospitals nationwide from 2013 to 2015 . Part of this study was previously published
[5], and part of the hospitals are listed in the Acknowledgements section.

Scrub typhus was confirmed either by eschar- or buffy coat-based polymerase chain reaction (PCR) or by a serologic assay. PCR targeting the variable domains I and II of the $56-\mathrm{kDa}$ antigen gene of O. tsutsugamushi was performed using a set of primers (forward: TTT CGA ACG TGT CTT TAA GC; reverse: ACA GAT GCA CTA TTA GGC AA; $1151 \mathrm{bp}$ ); the products were sequenced to match the reference genotypes, as described in a previous study [15]. The presence of fourfold or greater changes in the titers of the paired sera from an indirect immunofluorescence antibody assay (IFA) or a passive hemagglutination assay (GreenCross SangA; Yongin city, South Korea) was used as the positive serologic criteria. All sera from patients with confirmed scrub typhus were screened for the co-infection with SFTSV. SFTS is a reportable infectious disease to the Korea Centers for Disease Control and Prevention (KCDC) and all SFTSV infections were confirmed at the KCDC by detecting the $M$ segment gene of the SFTSV RNA using one-step reverse transcription (RT)-PCR as described in a previous study [16].

\section{Study design}

Baseline characteristics and clinical and laboratory parameters were compared between scrub typhus and SFTS to determine the differentiating factors. Scrub typhus was further subclassified into eschar-negative and -positive groups for comparison with SFTS. An SFTS prediction scoring tool was generated based on logistic regression analysis for SFTS. The baseline characteristics included demographic variables, comorbidities, site of infection, season of infection, duration from the onset of illness to first visit, duration of the hospital stay, and in-hospital mortality. The clinical parameters included commonly known symptoms and signs of both diseases such as headache, altered consciousness, cough, dyspnea, gastrointestinal manifestations, skin rash and the presence of a bite wound. The laboratory parameters included a complete blood count and chemistry, which can be easily obtained in primary care settings as a point-of-care testing. The worst values of the clinical and laboratory parameters within $24 \mathrm{~h}$ of the initial visit were used. The frequency of major complications during the clinical course was also compared.

Altered mentality was defined as a Glasgow coma scale score $<15$. Acute kidney injury was defined as serum creatinine levels $\geq 2.0 \mathrm{mg} / \mathrm{dL}$ and 1.5 times the baseline level [17]. Shock was defined as a mean arterial pressure $<65 \mathrm{mmHg}$. The categorical cut-offs for the comparison of some laboratory values such as thrombocytopenia (platelet count $<80,000 / \mathrm{mm}^{3}$ ), aspartate transaminase (AST) $\geq 400 \mathrm{IU} / \mathrm{L}$ and alanine transaminase $(\mathrm{ALT}) \geq 200 \mathrm{IU} / \mathrm{L}$ were chosen in view of 
their mean values in SFTS cases and their kinetics during the clinical course, as shown in a previous study [5]. Geographic location was divided into the western and eastern areas of South Korea. The western area included the Seoul metropolitan area and Gyeonggi, Chungcheong and Cholla provinces, which mostly consist of plain rice fields. The eastern area included Kangwon and Gyeongsang provinces, which mostly consist of hilly and mountainous areas.

\section{Statistical analysis}

Chi-square or Fisher's exact tests were used to analyze the categorical variables. T-tests or Mann-Whitney U-tests were used to compare the continuous variables. A multivariate logistic regression analysis was performed using the risk factors that were significantly $(P<0.05)$ associated with SFTS or scrub typhus in the univariate analysis and adjusted with the duration from the onset of illness to the initial presentation. The SFTS prediction scoring tool was generated using the logistic regression analysis for estimating odds ratios. The receiver operating characteristic curve was constructed for the scoring model (SPSS v20.0, Armonk, NY: IBM Corp.).

\section{Results}

A total of 362 patients were included in the analysis, including 255 patients with scrub typhus and 107 patients with SFTS. Eschar-positive scrub typhus accounted for $80.4 \%(205 / 255)$ and eschar-negative scrub typhus for $19.6 \%(50 / 255)$ of patients. Scrub typhus was confirmed by PCR in 153 patients and by serology in 102 patients. All patients with scrub typhus $(N=255)$ showed negative results for SFTSV in the sera. Compared to scrub typhus patients, patients with SFTS showed a higher median age (71 years), more comorbidities such as diabetes mellitus and hypertension, a greater tendency toward infection in the summer season, a greater tendency to be infected in the eastern area of South Korea, a shorter duration from the onset of illness to the first visit (median of 4 days), a longer duration of hospital stay (median of 10 days), and a higher case-fatality rate $(40.2 \%$ vs $0.4 \%$, respectively) (Table 1).

At the initial presentation, the overall subjective symptoms, except for gastrointestinal symptoms, were more prominent in patients with scrub typhus. Skin rash was predominantly present in scrub typhus cases (87.8 and $78.0 \%$ in eschar-positive and -negative scrub typhus respectively, vs $5.7 \%$ in SFTS). Bite wounds were present in $28.3 \%$ of SFTS patients. Altered mentality was more common in SFTS patients (27.9\%). In the subgroup comparisons, patients with eschar-negative scrub typhus presented fewer subjective symptoms. Fever was present in all the patients and was the initial chief problem leading to a hospital visit. The presence of skin rash (78.0\%) in eschar-negative scrub typhus was similar to the rate in eschar-positive scrub typhus $(P=0.110)$ (Table 2). Laboratory abnormalities were more prominent in SFTS. Leukopenia, thrombocytopenia, and an elevation of AST and lactate dehydrogenase (LDH) were more common in SFTS. C-reactive protein (CRP) was rarely elevated in SFTS (mean $1.24 \mathrm{mg} / \mathrm{dL}$ ) (Table 3). SFTS was associated with a disproportionately higher incidence of major complications during the hospitalization course such as decreased mentality (59.0\%), seizure (16.2\%), pneumonia (74.4\%), the need for mechanical ventilation (32.1\%), and acute kidney injury (21.5\%) (Table 4).

In the multivariate regression analysis, leukopenia (white blood cell count $<4000 / \mathrm{mm}^{3}$; odds ratio [OR] 30.13, $P<0.001$ ), thrombocytopenia (platelet count $<80,000 / \mathrm{mm}^{3}$; OR 19.73, $P<0.001)$ and low CRP $(<1 \mathrm{mg} / \mathrm{dL}$; OR 67.46, $P<0.001)$ were significantly predictive factors for SFTS compared with scrub typhus (Table 5). These 3 factors were consistently significant in a subgroup analysis compared with eschar-negative scrub typhus. As few laboratory results were significantly indicative of scrub typhus in comparison with SFTS in the univariate analysis, primarily only clinical variables were included in the multivariate analysis to identify the predictive factors for scrub typhus. In addition to the characteristic eschar and skin rash, headache was the only consistent risk factor for all cases of scrub typhus and the subgroup of eschar-negative scrub typhus.

A prediction scoring tool for the differential diagnosis of SFTS and scrub typhus was generated using the combination of those 3 parameters ( 1 point each for WBC count $<4000 / \mathrm{mm}^{3}$, platelet count $<80,000 / \mathrm{mm}^{3}$ and CRP value $<1 \mathrm{mg} / \mathrm{dL}$ ); the total score ranged from 0 to 3 . On the ROC curve obtained for this model, the optimal cut-off was $\geq 2$. A score $\geq 2$ had a sensitivity of $93.1 \%$ (95\% confidence interval [CI], 87.9-96.4\%) and a specificity of $96.1 \%$ (95\% CI, 93.8-97.6\%) for SFTS, with an ROC area under the curve of 0.972 (95\% CI, 0.952-0.990) (Table 6). In the eschar-negative scrub typhus subgroup, a score $\geq 2$ had a sensitivity of 93.1\% (95\% CI, 88.9-95.2\%) and a specificity of $93.9 \%$ (95\% CI, 85.0-98.3\%) for SFTS. In the subgroup of rash-negative scrub typhus, a score $\geq 2$ had a sensitivity of $93.1 \%$ (95\% CI, 89.0-95.2\%) and a specificity of $90.3 \%$ (95\% CI, 76.8-97.2\%) for SFTS (Table 6).

\section{Discussion}

A considerable number of SFTS cases occur during the epidemic season of scrub typhus in South Korea. In 2017, $86.7 \%(9132 / 10,528)$ of scrub typhus and $48.1 \%(131 / 272)$ of SFTS cases were officially reported to occur from September to November. Although the case-fatality rate of scrub typhus is low with antibiotic treatment, severe scrub typhus remains an unresolved issue $[18,19]$. The concurrent presence of the typical eschar with a compatible clinical manifestation makes the clinical diagnosis of scrub 
Table 1 Baseline characteristics of the subjects $(n=362)$

\begin{tabular}{|c|c|c|c|c|c|c|}
\hline \multirow[t]{3}{*}{ Variable } & \multicolumn{4}{|c|}{ Scrub typhus $(n=255)$} & \multirow{3}{*}{$\begin{array}{l}\text { SFTS } \\
n=107\end{array}$} & \multirow{3}{*}{$\begin{array}{l}P \\
\text { value }^{c}\end{array}$} \\
\hline & Eschar-positive & $P$ & Eschar-negative & \multirow{2}{*}{$\begin{array}{l}P \\
\text { value }^{b}\end{array}$} & & \\
\hline & \multicolumn{2}{|l|}{$n=205$} & $n=50$ & & & \\
\hline Age, years (median, IQR) & $60(49-71)$ & $<0.001$ & $64.5(51.2-70.7)$ & 0.004 & $71(61-78)$ & $<0.001$ \\
\hline Male gender, n (\%) & $81(39.5)$ & 0.044 & $22(44.0)$ & 0.387 & $55(51.4)$ & 0.054 \\
\hline \multicolumn{7}{|l|}{ Comorbidity } \\
\hline Diabetes mellitus & $19(9.3)$ & 0.027 & $5(10.0)$ & 0.200 & 19/106 (17.9) & 0.023 \\
\hline Hypertension & $44(21.5)$ & 0.030 & $13(26.0)$ & 0.395 & $35(32.7)$ & 0.039 \\
\hline CVA & $7(3.4)$ & 1.000 & $1(2.0)$ & 1.000 & $4(3.7)$ & 0.771 \\
\hline Congestive heart failure & $9(4.4)$ & 0.342 & $2(4.0)$ & 0.593 & $2(1.9)$ & 0.254 \\
\hline Chronic liver disease & $7(3.4)$ & 0.272 & $5(10.0)$ & 0.013 & $1(0.9)$ & 0.119 \\
\hline Asthma/COPD & $7(3.4)$ & 0.583 & $1(2.0)$ & 0.665 & $5(4.7)$ & 0.474 \\
\hline Solid tumor & $6(2.9)$ & 0.098 & $2(4.0)$ & 0.098 & 0 & 0.111 \\
\hline None & $126(61.5)$ & 0.121 & $26(52.0)$ & 0.969 & $56(52.3)$ & 0.202 \\
\hline \multicolumn{7}{|l|}{ Seasonal occurrence, n (\%) } \\
\hline Spring-summer (Mar-Aug) & 0 & $<0.001$ & 0 & $<0.001$ & $67(62.6)$ & $<0.001$ \\
\hline Autumn (Sep-Dec) & $205(100)$ & & $50(100)$ & & $40(37.4)$ & \\
\hline \multicolumn{7}{|l|}{ Geographical location } \\
\hline Western area & $167(81.5)$ & $<0.001$ & $42(84.0)$ & $<0.001$ & 36/104 (34.6) & $<0.001$ \\
\hline Eastern area & $38(18.5)$ & & $8(16.0)$ & & $68 / 104(65.4)$ & \\
\hline \multicolumn{7}{|l|}{ Duration, mean $( \pm S D)$, days } \\
\hline From onset of illness to admission & $6.63(3.918)$ & $<0.001$ & $6.38(5.103)$ & 0.006 & $4.39(3.66))$ & $<0.001$ \\
\hline Hospital stay & $6.26(11.927)$ & $<0.001$ & $6.18(3.757)$ & $<0.001$ & $12.07(9.57)$ & $<0.001$ \\
\hline Mortality, in-hospital & $1(0.5)$ & $<0.001$ & 0 & $<0.001$ & $43(40.2)$ & $<0.001$ \\
\hline
\end{tabular}

typhus obvious [20], but the poor sensitivity of diagnostic assays for scrub typhus and the presence of eschar-negative scrub typhus make the diagnosis uncertain in some patients. Given this diagnostic uncertainty combined with the potential of severe clinical form, a clinical prediction tool will be very useful to narrow the differential diagnosis for those requiring further urgent investigation [14].

Our prediction tool used 3 variables; leukopenia (WBC count $<4000 / \mathrm{mm}^{3}$ ), thrombocytopenia (platelet count $\left.<80,000 / \mathrm{mm}^{3}\right)$ and low CRP $(<1 \mathrm{mg} / \mathrm{dL})$, which could be obtained from routine basic laboratory blood tests and are readily applicable in primary care settings. The cut-off level of thrombocytopenia as platelet count of $80,000 / \mathrm{mm}^{3}$ was determined by considering the distribution of platelet counts among the study subjects and the kinetics of initially persistent thrombocytopenia in SFTS [5]. One study previously proposed a similar scoring system using 4 variables: altered mental status, leukopenia, prolonged activated partial thromboplastin time and normal C-reactive protein [21]. In our study, 'altered mental status' was not a significant factor to be incorporated into the prediction analysis. We objectively assessed mental status using the Glasgow coma scale, which is one of the basic tools used in critical care. We did not include the coagulation panels in the initial comparison because we do not routinely check coagulation panels during the investigation of possible scrub typhus cases.

A larger sample size might have led to different results. Our study also included a relatively larger number of eschar-negative scrub typhus cases, which causes diagnostic challenges and requires a clinical decision to guide further diagnostic evaluations. We adjusted the comparison with the duration from the onset of illness to the initial presentation because the kinetics of clinical variables are closely time-dependent. The prediction tool worked similarly in all subgroups of scrub typhus (Table 6). The data collection from multiple 
Table 2 Clinical symptom and sign at the initial presentation $(n=362)$

\begin{tabular}{|c|c|c|c|c|c|c|}
\hline \multirow[t]{3}{*}{ Variable } & \multicolumn{4}{|c|}{ Scrub typhus $(n=255)$} & \multirow{3}{*}{$\begin{array}{l}\text { SFTS } \\
n=107\end{array}$} & \multirow{3}{*}{$\begin{array}{l}P \\
\text { value }^{c}\end{array}$} \\
\hline & Eschar-positive & \multirow{2}{*}{$\begin{array}{l}P \\
\text { value }^{a}\end{array}$} & Eschar-negative & \multirow{2}{*}{$\begin{array}{l}P \\
\text { value }^{b}\end{array}$} & & \\
\hline & $n=205$ & & $n=50$ & & & \\
\hline Fever & $205(100)$ & $<0.001$ & $50(100)$ & 0.017 & 95/106 (89.6) & $<0.001$ \\
\hline Headache & $179(87.3)$ & $<0.001$ & $30(60.0)$ & $<0.001$ & 26/105 (24.8) & $<0.001$ \\
\hline Myalgia & $172(83.9)$ & $<0.001$ & $29(58.0)$ & 0.794 & $58 / 104(55.8)$ & $<0.001$ \\
\hline Conjunctival injection & $78(38.0)$ & $<0.001$ & $6(12.0)$ & 0.215 & 6/98 (6.1) & $<0.001$ \\
\hline Sore throat & $74(36.1)$ & $<0.001$ & $11(22.0)$ & 0.005 & $7 / 105(6.7)$ & $<0.001$ \\
\hline Cough & $76(37.1)$ & $<0.001$ & $15(30.0)$ & 0.004 & $12 / 105(11.4)$ & $<0.001$ \\
\hline Dyspnea & $47(22.9)$ & 0.004 & $9(18.0)$ & 0.138 & 10/104 (9.6) & 0.006 \\
\hline Nausea/vomiting & $81(39.5)$ & 0.463 & $14(28.0)$ & 0.370 & $37 / 105(35.2)$ & 0.718 \\
\hline Abdominal pain & $58(28.3)$ & 0.019 & $9(18.0)$ & 0.778 & 17/105 (16.2) & 0.033 \\
\hline Arthralgia & $62(30.2)$ & $<0.001$ & $4(8.0)$ & 0.429 & $5 / 104(4.8)$ & $<0.001$ \\
\hline Skin rash & $180(87.8)$ & $<0.001$ & $39(78.0)$ & $<0.001$ & $6 / 105(5.7)$ & $<0.001$ \\
\hline Presence of bite wound & $205(100)$ & $<0.001$ & $0(0)$ & $<0.001$ & $30 / 106(28.3)$ & $<0.001$ \\
\hline Altered mentality & $11(5.4)$ & $<0.001$ & $5(10.0)$ & 0.012 & 29/104 (27.9) & $<0.001$ \\
\hline Shock (MAP < 65 mmHg) & $15(7.3)$ & 0.085 & $4(8.0)$ & 0.427 & 14/105 (13.3) & 0.079 \\
\hline
\end{tabular}

Abbreviations: SFTS severe fever with thrombocytopenia syndrome, MAP mean arterial pressure

a $P$ value when compared to SFTS

${ }^{\mathrm{b}} P$ value when compared to SFTS

${ }^{c} P$ value when compared to all scrub typhus

hospitals in major endemic areas of the two diseases might strengthen the generalizability of our study.

In a binary comparison of clinical parameters, scrub typhus tended to present with more subjective manifestations, whereas SFTS showed more laboratory abnormalities. In a subgroup comparison of eschar-negative scrub typhus with SFTS, skin rash and headache were significantly indicative of scrub typhus. As the skin rash in scrub typhus is a characteristic maculopapular type that is clinically distinct from that of SFTS [20], the presence of a maculopapular skin rash itself has diagnostic value if the clinician is sufficiently experienced. However, approximately $10 \%$ of patients with scrub typhus are reported to lack the typical skin rash [22, 23]. Therefore, scrub typhus without eschar and a skin rash poses a further diagnostic challenge. In our study, $12.2 \%$ of eschar-positive and $22.0 \%$ of eschar-negative scrub typhus patients had no skin rash. The rate of escharnegative scrub typhus has been reported to be approximately $10 \%$, although a skilled physician may observe a different rate [22, 23].

Reports regarding co-infection of both scrub typhus and SFTS are limited. In a recent report, $23.0 \%$ of patients clinically suspected of scrub typhus were SFTS-positive [24]. There was a case report of co-infection diagnosed by PCR [25]. In another report, however, none of the 38 patients with scrub typhus were SFTS-positive, whereas one of 21 patients with SFTS was serologically suggestive of scrub typhus. Thus, the clinical evidence on the possibility of co-infection is not solid, and further monitoring is necessary. From an ecological perspective, given the vectors of the two diseases, co-infection is not likely. Although the epidemic seasons overlap and there is a risk of simultaneously acquiring the two diseases during outdoor activity, they do not share vectors, and the ecologies of their vectors differs. Phenotypically, scrub typhus is highly prevalent in the rice field areas of western and southwestern South Korea [26], whereas the incidence of SFTS is low in this area, consistent with the low SFTSV infection rate in ticks. Conversely, the incidence of SFTS is high in the eastern and southeastern mountainous area of South Korea [5, 27].

The causative agent of scrub typhus, O. tsutsugamushi, is transmitted by trombiculid chigger mites. The causative Trombiculidae have a nationwide distribution in South Korea [28, 29]. Although the larvae of trombiculid mites can parasitize most animals, rodents and some other small mammals are their primary hosts [30,31]. The prevalence and abundance of chigger mites on small mammals are much higher in cultivated flatland landscapes [32]. Meanwhile, Haemaphysalis longicornis is the predominant vector for SFTSV, but other tick species such as $H$. flava, Amblyomma testudinarium and Ixodes nipponensis can also carry SFTSV in South Korea [27, 33, 34]. $H$. longicornis is able to transmit SFTSV via both transovarial and transstadial modes [35]. H. longicornis is widely distributed in Australia, New Zealand, Korea, Japan and China [36]. Larger mammals, such as rabbits, badgers, 
Table 3 Laboratory findings at the initial presentation $(n=362)$

\begin{tabular}{|c|c|c|c|c|c|c|}
\hline \multirow[t]{2}{*}{ Variable } & \multicolumn{4}{|c|}{ Scrub typhus $(n=255)$} & \multirow{2}{*}{$\begin{array}{l}\text { SFTS } \\
n=107\end{array}$} & \multirow{2}{*}{$\begin{array}{l}P \\
\text { value }^{c}\end{array}$} \\
\hline & $\begin{array}{l}\text { Eschar-positive } \\
n=205\end{array}$ & $\begin{array}{l}P \\
\text { value }^{a}\end{array}$ & $\begin{array}{l}\text { Eschar-negative } \\
n=50\end{array}$ & $\begin{array}{l}P \\
\text { value }^{b}\end{array}$ & & \\
\hline White blood cells, $/ \mathrm{mm}^{3}$ & $6909 \pm 3755$ & $<0.001$ & $8426 \pm 4207$ & $<0.001$ & $2317 \pm 1930$ & $<0.001$ \\
\hline Leukopenia $\left(<4000 / \mathrm{mm}^{3}\right)$, \% & $42(20.5)$ & $<0.001$ & $5(10.0)$ & $<0.001$ & $97 / 106(91.5)$ & $<0.001$ \\
\hline Hemoglobin, g/dL & $12.8 \pm 1.8$ & 0.007 & $12.1 \pm 1.6$ & $<0.001$ & $13.4 \pm 1.7$ & 0.001 \\
\hline Anemia $(<11$ g/dL) & $22(10.7)$ & 0.532 & $11(22.0)$ & 0.019 & 9/106 (8.5) & 0.230 \\
\hline Platelet $\left(\times 10^{3} / \mathrm{mm}^{3}\right)$ & $133 \pm 47$ & $<0.001$ & $161 \pm 89$ & $<0.001$ & $61 \pm 30$ & $<0.001$ \\
\hline Thrombocytopenia ${ }^{d}, \%$ & $21(10.2)$ & $<0.001$ & $5(10.0)$ & $<0.001$ & 83/106 (78.3) & $<0.001$ \\
\hline Serum albumin, $\mathrm{d} / \mathrm{dL}$ & $3.5 \pm 0.6$ & 0.575 & $3.07 \pm 0.59$ & 0.031 & $3.4 \pm 0.6$ & 0.851 \\
\hline Total bilirubin, mg/dL & $0.93 \pm 1.14$ & $<0.001$ & $0.64 \pm 0.66$ & 0.034 & $0.40 \pm 0.61$ & $<0.001$ \\
\hline AST, IU/L & $108 \pm 107$ & $<0.001$ & $170 \pm 277$ & 0.007 & $381 \pm 505$ & $<0.001$ \\
\hline$\geq 400 \mathrm{IU} / \mathrm{L}$ & $4 / 204(2.0)$ & $<0.001$ & $4(8.0)$ & 0.001 & $33 / 106(31.1)$ & $<0.001$ \\
\hline$A L T, I U / L$ & $96 \pm 108$ & 0.026 & $138 \pm 192$ & 0.824 & $131 \pm 159$ & 0.135 \\
\hline$\geq 200 \mathrm{IU} / \mathrm{L}$ & $16 / 204(7.8)$ & 0.002 & $9(18.0)$ & 0.789 & 21/106 (19.8) & 0.010 \\
\hline Lactate dehydrogenase, IU/L & $700 \pm 423$ & $<0.001$ & $761 \pm 393$ & 0.014 & $1615 \pm 2159$ & $<0.001$ \\
\hline$\geq 800 \mathrm{IU} / \mathrm{L}$ & 48/183 (26.2) & $<0.001$ & $14 / 40(35.0)$ & 0.021 & $55 / 97(56.7)$ & $<0.001$ \\
\hline Serum creatinine, mg/dL & $1.04 \pm 0.63$ & 0.634 & $1.14 \pm 0.70$ & 0.567 & $1.08 \pm 0.51$ & 0.841 \\
\hline$\geq 2.0 \mathrm{mg} / \mathrm{dL}$ & $5 / 203(2.5)$ & 1.000 & $4(8.0)$ & 0.086 & 2/105 (1.9) & 0.519 \\
\hline Hypokalemia (<3.5 mmol/L) & $52 / 204(25.5)$ & 0.155 & $12 / 49(24.5)$ & 0.372 & 19/104 (18.3) & 0.153 \\
\hline C-reactive protein, mg/dL & $7.27 \pm 5.50$ & $<0.001$ & $9.82 \pm 7.41$ & $<0.001$ & $1.24 \pm 2.64$ & $<0.001$ \\
\hline$<1 \mathrm{mg} / \mathrm{dL}$ & 7/182 (3.8) & $<0.001$ & $1 / 49(2.0)$ & $<0.001$ & $66 / 102(64.7)$ & $<0.001$ \\
\hline
\end{tabular}

Abbreviations: SFTS severe fever with thrombocytopenia syndrome, AST aspartate aminotransferase, $A L T$ alanine aminotransferase

ap value when compared to SFTS

${ }^{\mathrm{b}} P$ value when compared to SFTS

${ }^{c} P$ value when compared to all scrub typhus

${ }^{\mathrm{d}}$ Thrombocytopenia, $<80 \times 10^{3} / \mathrm{mm}^{3}$

deer and wild boars, rather than rodents, have been suggested to be the principal hosts for this tick species [36]. The differences between the main hosts and habitats of the vectors makes their simultaneous exposure less likely. However, the enhanced intrusion of wild animals into cultivated farmland areas and serologic evidence of SFTSV infection in domestic animals may indicate the exposure risk to dual vectors $[37,38]$. The case-fatality trend of scrub typhus also deserves to be mentioned for indirect evidence of co-infection. Although there are approximately 10,000 cases of scrub typhus annually in South Korea, the recent annual mortality was stable in the range

Table 4 Major complications during the clinical course $(n=362)$

\begin{tabular}{|c|c|c|c|c|c|c|}
\hline \multirow[t]{2}{*}{ Variable } & \multicolumn{4}{|c|}{ Scrub typhus $(n=255)$} & \multirow{2}{*}{$\begin{array}{l}\text { SFTS } \\
n=107\end{array}$} & \multirow{2}{*}{$\begin{array}{l}P \\
\text { value }^{c}\end{array}$} \\
\hline & $\begin{array}{l}\text { Eschar-positive } \\
n=205\end{array}$ & $\begin{array}{l}P \\
\text { value }^{a}\end{array}$ & $\begin{array}{l}\text { Eschar-negative } \\
n=50\end{array}$ & $\begin{array}{l}P \\
\text { value }^{b}\end{array}$ & & \\
\hline \multicolumn{7}{|l|}{ CNS involvement } \\
\hline Altered mentality $(\mathrm{GCS}<15)$ & $14 / 199(7.0)$ & $<0.001$ & $8(16.0)$ & $<0.001$ & $62 / 105(59.0)$ & $<0.001$ \\
\hline Seizure & 0/199 (0) & $<0.001$ & $0(0)$ & 0.002 & $17 / 105(16.2)$ & $<0.001$ \\
\hline \multicolumn{7}{|l|}{ Lung involvement } \\
\hline Mechanical ventilation & $5(2.4)$ & $<0.001$ & $2(4.0)$ & $<0.001$ & 34/106 (32.1) & $<0.001$ \\
\hline \multicolumn{7}{|l|}{ Renal involvement } \\
\hline Acute kidney injury & $5(2.4)$ & $<0.001$ & $5(10.0)$ & 0.080 & $23(21.5)$ & $<0.001$ \\
\hline
\end{tabular}

Abbreviations: SFTS severe fever with thrombocytopenia syndrome, CNS central nervous system, GCS Glasgow coma scale

${ }^{a} P$ value when compared to SFTS

${ }^{\mathrm{b}} P$ value when compared to SFTS

${ }^{c} P$ value when compared to all scrub typhus 
Table 5 Multivariate analysis for the predictive factors of SFTS compared to those of eschar-positive and negative scrub typhus

\begin{tabular}{lll}
\hline Variables & Odds ratio $(95 \% \mathrm{Cl})$ & $P$ value \\
\hline Age & $1.03(0.98-1.09)$ & 0.145 \\
Male gender & $2.09(0.58-7.44)$ & 0.255 \\
Diabetes mellitus & $2.53(0.36-17.43)$ & 0.345 \\
Hypertension & $0.64(0.12-3.28)$ & 0.593 \\
Duration, from onset of illness to initial visit & $0.80(0.68-0.94)$ & 0.008 \\
Altered mentality & $2.31(0.27-19.33)$ & 0.439 \\
Shock & $1.72(0.23-12.68)$ & 0.592 \\
Leukopenia (WBC $\left.<4000 / \mathrm{mm}^{3}\right)$ & $30.13(6.08-149.22)$ & $<0.001$ \\
Thrombocytopenia $\left(<80,000 / \mathrm{mm}^{3}\right)$ & $19.73(4.60-84.58)$ & $<0.001$ \\
AST $\geq 400 \mathrm{IU} / \mathrm{L}$ & $8.33(0.39-176.07)$ & 0.173 \\
ALT $\geq 200 \mathrm{IU} / \mathrm{L}$ & $0.36(0.03-4.07)$ & 0.411 \\
LDH $\geq 800 \mathrm{IU} / \mathrm{L}$ & $3.05(0.65-14.15)$ & 0.154 \\
C-reactive protein $<1 \mathrm{mg} / \mathrm{dL}^{2}$ & $67.46(14.29-318.30)$ & $<0.001$ \\
\hline Abbrit
\end{tabular}

Abbreviations: SFTS severe fever with thrombocytopenia syndrome, $C l$ confidence interval, WBC white blood cell, AST aspartate aminotransferase, ALT alanine aminotransferase, $L D H$ lactate dehydrogenase

of 11-13 cases (http://www.cdc.go.kr/npt). This suggests that co-infection is rare, considering the high mortality and rising incidence of SFTS.

The magnitude of the prevalence of SFTS and the need for intense differential vigilance in the community must be further investigated. The seroprevalence for SFTSV antibodies in South Korea were reported to be 2.7 to $7.7 \%$ in rural area and $1.9 \%$ in urban areas in small-scale studies $[39,40]$. SFTS is already endemic throughout South Korea, and the rapidly increasing trend of its incidence is obvious [5]. The severity and poor prognosis of SFTS demand accurate initial clinical triage. Human granulocytic anaplasmosis and human monocytotrophic ehrlichiosis which have similar clinical presentations are also important differential diseases. Hence, we suggest a clinical decision algorithm based on our findings as follows. In an atypical febrile disease during the epidemic season of scrub typhus, the empirical administration of doxycycline is desirable. Positive findings of eschar or a maculopapular skin rash on physical examination are strongly suggestive of scrub typhus and are an indication for maintaining doxycycline treatment. If there is no eschar or maculopapular rash, the calculation of the prediction score in our study may guide the degree of suspicion for SFTS. Until confirming the presence of SFTS, continuing doxycycline may be clinically useful to cover eschar- and skin rashnegative scrub typhus, anaplasmosis, and ehrlichiosis, which all respond to doxycycline.

This study has several limitations. First, the prediction scoring tool was evaluated only in comparison with scrub typhus. Further performance assessment in general febrile patients may be useful to demonstrate the utility of this tool for clinically identifying SFTS. Second, although the SFTS data were primarily obtained from intensive clinical care settings, the retrospective nature of data collection might lead to a bias in contrast to the prospective collection of scrub typhus cases. In addition, only viremic SFTS cases were included, which might exclude mild cases.

Table 6 Diagnostic performance of the SFTS prediction scoring system

\begin{tabular}{|c|c|c|c|c|}
\hline SFTS score & Sensitivity $(95 \% \mathrm{Cl})$ & Specificity $(95 \% \mathrm{Cl})$ & Positive likelihood ratio (95\% Cl) & Negative likelihood ratio $(95 \% \mathrm{Cl})$ \\
\hline \multicolumn{5}{|c|}{ A. SFTS vs. eschar-positive and -negative scrub typhus } \\
\hline$\geq 2$ & $0.931(0.879-0.964)$ & $0.961(0.938-0.976)$ & $23.905(14.211-39.547)$ & $0.071(0.037-0.128)$ \\
\hline 3 & $0.422(0.376-0.431)$ & $0.996(0.976-1.000)$ & $97.382(15.379-1903.086)$ & $0.581(0.569-0.640)$ \\
\hline \multicolumn{5}{|c|}{ B. SFTS vs. eschar-negative scrub typhus } \\
\hline$\geq 2$ & $0.931(0.889-0.952)$ & $0.939(0.850-0.983)$ & $15.212(5.925-54.850)$ & $0.073(0.048-0.131)$ \\
\hline 3 & $0.422(0.382-0.422)$ & $1.000(0.918-1.000)$ & NA & $0.578(0.578-0.673)$ \\
\hline \multicolumn{5}{|c|}{ C. SFTS vs. rash-negative scrub typhus } \\
\hline$\geq 2$ & $0.931(0.890-0.952)$ & $0.903(0.768-0.972)$ & $9.624(3.836-34.539)$ & $0.076(0.049-0.143)$ \\
\hline 3 & $0.422(0.384-0.422)$ & $1.000(0.875-1.000)$ & NA & $0.578(0.578-0.705)$ \\
\hline
\end{tabular}

Abbreviations: SFTS severe fever with thrombocytopenia syndrome, $\mathrm{Cl}$ confidence interval, NA not available 
However, our concentration on moderate to severe cases might have greater clinical impacts in practice. Third, we did not evaluate other parameters such as activated partial thromboplastin time or ferritin, which have also been suggested as useful markers for SFTS, because the previous scrub typhus studies did not include those variables [21, 41]. However, these laboratory variables are not readily available at a point of care or are not necessary for usual clinical practices in the primary care settings where the confirmatory assays for both diseases are not available. Lastly, we used several cohorts of different time points. As there is no evidence that the clinical features of scrub typhus and SFTS have changed respectively, and we have used a same methodology, mixing of cohorts might not lead to the significant inhomogeneity.

\section{Conclusions}

We suggested a clinical prediction scoring tool for SFTS in comparison with scrub typhus that consists of 3 variables: leukopenia (WBC count $<4000 / \mathrm{mm}^{3}$ ), thrombocytopenia (platelet count $<80,000 / \mathrm{mm}^{3}$ ) and low CRP $(<1 \mathrm{mg} / \mathrm{dL})$. It is a simple and readily applicable tool that can be used in primary care settings. It will be useful for differentiating between SFTS and eschar- and skin rash-negative scrub typhus. We also showed an in-depth comparison of SFTS and scrub typhus to better understand the clinical features of both diseases. This tool may also be used to screen out SFTS in areas where SFTS has not yet been reported but is geographically capable of existing because of proximity to the endemic countries.

\section{Abbreviations}

ALT: Alanine transaminase; AST: Aspartate transaminase; Cl: Confidence interval; CRP: C-reactive protein; IFA: Indirect immunofluorescence antibody assay; KCDC: Korea Centers for Disease Control and Prevention; LDH: Lactate dehydrogenase; OR: Odds ratio; PCR: Polymerase chain reaction; RT: Reverse transcription; SFTS: Severe fever with thrombocytopenia syndrome; SFTSV: SFTS virus

\section{Acknowledgements}

We thank our collaborators for collecting the data: Jacob Lee (Hallym University Medical Center, Seoul), Eun Hee Song (GangNeung Asan Hospital, GangNeung), Ki-Ho Park (Kyung Hee University Hospital, Seoul), Joon Young Song (Korea University Guro Hospital, Seoul), Dae Won Park (Korea University Ansan Hospital), Young Kyung Yoon (Korea University Anam Hospital), Hyun Hee Kwon (Daegu Catholic University Medical Center, Daegu), Cheol-In Kang (Samsung Medical Center, Seoul), Yu Mi Wi (Samsung Changwon Hospital, Changwon), Seong-Heon Wie (St. Vincent's Hospital, Suwon), Sang Hoon Han (Severance Hospital, Seoul), Yong Kyun Cho (Gachon University Gil Medical Center, Incheon), Jin-Soo Lee (Inha University Hospital, Incheon), Yoon Hee Jun (Cheju Halla Hospital, Jeju), Min Hee Lim (Changwon Fatima Hospital, Changwon), Kyung-Wook Hong (Hallym University Sacred Heart Hospital, Chuncheon), Moon-Hyun Chung (Hanmaeum Hospital, Jeju), Jae Myung Kang (Sunlin Hospital, Pohang) and Sung min Kiem (Inje University Paik Hospital, Busan), all from the Republic of Korea.

\section{Funding}

This study was supported by grants from the Korea Centers for Disease Control and Prevention (2015-E24001-00, http://www.cdc.go.kr; MdO), the Seoul National University Hospital Research Fund (04-2009-610, snuh.org;
SWP) and the Seoul Metropolitan Government Seoul National University Boramae Medical Center (03-2018-14, http://www.brmh.org; SWP). The funders had no role in study design, data collection and analysis, decision to publish, or preparation of the manuscript.

\section{Availability of data and materials}

The datasets used and/or analyzed during this study are available from the corresponding author on reasonable request.

\section{Authors' contributions}

SWP and MdO designed and had funding sources for the study. CSL, JHK, IGB, CSM, YGK, BNK, JHL, SYR, HCJ, JH, JBJ, YJ, HHC, YKK, JHH, YSK, HWJ, KHS, WBP and ESK substantially contributed to the acquisition, analysis and interpretation of data. SWP, JHK and MdO have drafted the work and finalized the manuscript. All authors critically read, revised and approved the final version of the manuscript, took part sufficiently in the work to take public responsibility for all aspects of the work in ensuring that questions related to the accuracy or integrity of any part of the work were appropriately investigated and resolved.

\section{Ethics approval and consent to participate}

This study was approved by the institutional review board of Boramae Medical Center (10-2018-68), which waived the need of obtaining consent from the patients. Personal information was de-identified before data retrieval and the anonymized data were processed by different analyzers. All clinical investigations were conducted according to the principles expressed in the Declaration of Helsinki.

\section{Consent for publication}

Not applicable.

\section{Competing interests}

The authors declare that they have no competing interests.

\section{Publisher's Note}

Springer Nature remains neutral with regard to jurisdictional claims in published maps and institutional affiliations.

\section{Author details}

${ }^{1}$ Department of Internal Medicine, Seoul National University College of Medicine, 103 Daehak-ro, Jongno-gu, Seoul 03080, the Republic of Korea. ${ }^{2}$ Department of Internal Medicine, Boramae Medical Center, Seoul, Republic of Korea. ${ }^{3}$ Department of Internal Medicine, Chonbuk National University Medical School, Jeonju, Republic of Korea. ${ }^{4}$ Department of Internal Medicine, Gyeongsang National University School of Medicine, Jinju, Republic of Korea. ${ }^{5}$ Department of Internal Medicine, Inje University College of Medicine, Busan, Republic of Korea. ${ }^{6}$ Department of Internal Medicine, Wonkwang University School of Medicine, Iksan, Republic of Korea. ${ }^{7}$ Department of Internal Medicine, Keimyung University Dongsan Medical Center, Daegu, Republic of Korea. ${ }^{8}$ Department of Infectious Diseases, Chonnam National University Medical School, Gwangju, Republic of Korea. ${ }^{9}$ Department of Internal Medicine, Yeungnam University College of Medicine, Daegu, Republic of Korea. ${ }^{10}$ Department of Internal Medicine, Ulsan University Hospital, University of Ulsan College of Medicine, Ulsan, Republic of Korea.

${ }^{11}$ Department of Internal Medicine, Hallym University Sacred Heart Hospital, Anyang, Republic of Korea. ${ }^{12}$ Department of Internal Medicine, School of Medicine, Kyungpook National University Kyungpook National University Hospital, Daegu, Republic of Korea. ${ }^{13}$ Department of Internal Medicine, Yonsei University Wonju College of Medicine, Wonju, Republic of Korea. ${ }^{14}$ Department of Internal Medicine, Chungnam National University School of Medicine, Daejeon, Republic of Korea. ${ }^{15}$ Department of Internal Medicine, Chungbuk National University College of Medicine, Cheongju, Republic of Korea. ${ }^{16}$ Department of Internal Medicine, Seoul National University Bundang Hospital, Seongnam, Republic of Korea. 
Received: 23 November 2018 Accepted: 1 February 2019

\section{Published online: 19 February 2019}

\section{References}

1. Yu XJ, Liang MF, Zhang SY, Liu Y, Li JD, Sun YL, et al. Fever with thrombocytopenia associated with a novel bunyavirus in China. N Engl J Med. 2011:364(16):1523-32

2. Kim KH, Yi J, Kim G, Choi SJ, Jun Kl, Kim NH, et al. Severe fever with thrombocytopenia syndrome, South Korea, 2012. Emerg Infect Dis. 2013; 19(11):1892-4

3. Takahashi T, Maeda K, Suzuki T, Ishido A, Shigeoka T, Tominaga T, et al. The first identification and retrospective study of severe fever with thrombocytopenia syndrome in Japan. J Infect Dis. 2014;209(6):816-27.

4. Jia B, Yan X, Chen Y, Wang G, Liu Y, Xu B, et al. A scoring model for predicting prognosis of patients with severe fever with thrombocytopenia syndrome. PLoS Negl Trop Dis. 2017;11(9):e0005909.

5. Choi SJ, Park SW, Bae IG, Kim SH, Ryu SY, Kim HA, et al. Severe fever with thrombocytopenia syndrome in South Korea, 2013-2015. PLoS Negl Trop Dis. 2016;10(12):e0005264.

6. Gokuden M, Fukushi S, Saijo M, Nakadouzono F, Iwamoto Y, Yamamoto M, et al. Low seroprevalence of severe fever with thrombocytopenia syndrome virus antibodies in individuals living in an endemic area in Japan. Jpn J Infect Dis. 2018;71(3):225-8.

7. Reece LM, Beasley DW, Milligan GN, Sarathy W, Barrett AD. Current status of severe fever with thrombocytopenia syndrome vaccine development. Curr Opin Virol. 2018:29:72-8

8. World Health Organization. 2017 Annual review of diseases prioritized under the research and development blueprint, 2017. http://www.who.int/ blueprint/what/research-development/2017-Prioritization-Long-Report pdf?ua=1. Accessed 30 Oct 2018

9. Bonell A, Lubell Y, Newton PN, Crump JA, Paris DH. Estimating the burden of scrub typhus: a systematic review. PLoS Negl Trop Dis. 2017; 11(9):e0005838

10. Oh WS, Heo ST, Kim SH, Choi WJ, Han MG, Kim JY. Plasma exchange and ribavirin for rapidly progressive severe fever with thrombocytopenia syndrome. Int J Infect Dis. 2014;18:84-6.

11. Park SY, Choi WY, Chong YP, Park SW, Wang EB, Lee WJ, et al. Use of plasma therapy for severe fever with thrombocytopenia syndrome encephalopathy. Emerg Infect Dis. 2016;22(7):1306-8

12. Kim UJ, Kim DM, Ahn JH, Kang SJ, Jang HC, Park KH, et al. Successful treatment of rapidly progressing severe fever with thrombocytopenia syndrome with neurological complications using intravenous immunoglobulin and corticosteroid. Antivir Ther. 2016;21:637-40.

13. Kim WY, Choi W, Park SW, Wang EB, Lee WJ, Jee Y, et al. Nosocomial transmission of severe fever with thrombocytopenia syndrome in Korea. Clin Infect Dis. 2015;60(11):1681-3.

14. Saraswati K, Day NPJ, Mukaka M, Blacksell SD. Scrub typhus point-ofcare testing: a systematic review and meta-analysis. PLoS Negl Trop Dis. 2018;12(3):e0006330.

15. Park SW, Lee CK, Kwak YG, Moon C, Kim BN, Kim ES, et al. Antigenic drift of Orientia tsutsugamushi in South Korea as identified by the sequence analysis of a 56-kDa protein-encoding gene. Am J Trop Med Hyg. 2010;83(4):930-5.

16. Shin J, Kwon D, Youn SK, Park JH. Characteristics and factors associated with death among patients hospitalized for severe fever with thrombocytopenia syndrome, South Korea, 2013. Emerg Infect Dis. 2015;21(10):1704-10.

17. Initiative KDIGO. KDIGO Clinical practice guideline for acute kidney injury. Kidney Int Suppl. 2012;2(1):1-138.

18. Kim DM, Kim SW, Choi SH, Yun NR. Clinical and laboratory findings associated with severe scrub typhus. BMC Infect Dis. 2010;10:108.

19. Park SW, Lee CS, Lee CK, Kwak YG, Moon C, Kim BN, et al. Severity predictors in eschar-positive scrub typhus and role of serum osteopontin. Am J Trop Med Hyg. 2011;85(5):924-30.

20. Lee CS, Hwang JH. Images in clinical medicine. Scrub typhus. N Engl J Med. 2015;373(25):2455

21. Kim MC, Chong YP, Lee SO, Choi SH, Kim YS, Woo JH, et al. Differentiation of severe fever with thrombocytopenia syndrome from scrub typhus. Clin Infect Dis. 2018;66(10):1621-4

22. Liu YX, Feng D, Suo J, Xing YB, Liu G, Liu LH, et al. Clinical characteristics of the autumn-winter type scrub typhus cases in south of Shandong province, northern China. BMC Infect Dis. 2009;9:82.
23. Ogawa M, Hagiwara T, Kishimoto T, Shiga S, Yoshida Y, Furuya Y, et al. Scrub typhus in Japan: epidemiology and clinical features of cases reported in 1998. Am J Trop Med Hyg. 2002;67:162-5.

24. Wi YM, Woo HI, Park D, Lee $\mathrm{KH}$, Kang Cl, Chung DR, et al. Severe fever with thrombocytopenia syndrome in patients suspected of having scrub typhus. Emerg Infect Dis. 2016;22(11):1992-5.

25. Yoo JR, Heo ST, Kang JH, Park D, Kim JS, Bae JH, et al. Mixed infection with severe fever with thrombocytopenia syndrome virus and two genotypes of scrub typhus in a patient, South Korea, 2017. Am J Trop Med Hyg. 2018;99(2):287-90.

26. Park SW, Ha NY, Ryu B, Bang JH, Song H, Kim Y, et al. Urbanization of scrub typhus disease in South Korea. PLoS Negl Trop Dis. 2015;9(5):e0003814.

27. Park SW, Song BG, Shin EH, Yun SM, Han MG, Park MY, et al. Prevalence of severe fever with thrombocytopenia syndrome virus in Haemaphysalis longicornis ticks in South Korea. Ticks Tick Borne Dis. 2014;5(6):975-7.

28. Korea CDC. Determination of vector species of tsutsugamushi disease and their geographical distribution in Korea. Public Health Weekly Rep. 2009;50(2):1-5.

29. Roh JY, Song BG, Park WI, Shin EH, Park C, Park MY, et al. Coincidence between geographical distribution of Leptotrombidium scutellare and scrub typhus incidence in South Korea. PLoS One. 2014;9(12):e113193.

30. Guo XG, Speakman JR, Dong WG, Men XY, Qian TJ, Wu D, et al. Ectoparasitic insects and mites on Yunnan red-backed voles (Eothenomys miletus) from a localized area in southwest China. Parasitol Res. 2013;112(10):3543-9.

31. Zhan YZ, Guo XG, Speakman JR, Zuo XH, Wu D, Wang QH, et al. Abundances and host relationships of chigger mites in Yunnan Province, China. Med Vet Entomol. 2013;27(2):194-202.

32. Peng PY, Guo XG, Jin DC, Dong WG, Qian TJ, Qin F, et al. Landscapes with different biodiversity influence distribution of small mammals and their ectoparasitic chigger mites: a comparative study from southwest China. PLoS One. 2018;13(1):e0189987.

33. Yun SM, Lee WG, Ryou J, Yang SC, Park SW, Roh JY, et al. Severe fever with thrombocytopenia syndrome virus in ticks collected from humans, South Korea, 2013. Emerg Infect Dis. 2014;20(8):1358-61.

34. Oh SS, Chae JB, Kang JG, Kim HC, Chong ST, Shin JH, et al. Detection of severe fever with thrombocytopenia syndrome virus from wild animals and Ixodidae ticks in the republic of Korea. Vector Borne Zoonotic Dis. 2016;16(6):408-14.

35. Zhuang L, Sun Y, Cui XM, Tang F, Hu JG, Wang LY, et al. Transmission of severe fever with thrombocytopenia syndrome virus by Haemaphysalis longicornis ticks, China. Emerg Infect Dis. 2018;24(5):868-71.

36. Zheng H, Yu Z, Zhou L, Yang X, Liu J. Seasonal abundance and activity of the hard tick Haemaphysalis longicornis (Acari: Ixodidae) in North China. Exp Appl Acarol. 2012:56(2):133-41.

37. Guoyu N, Jiandong L, Mifang L, Xiaolin J, Mei J, Haiying Y, et al. Severe fever with thrombocytopenia syndrome virus among domesticated animals, China. Emerg Infect Dis. 2013;19(5):756.

38. Kang JG, Oh SS, Jo YS, Chae JB, Cho YK, Chae JS. Molecular detection of severe fever with thrombocytopenia syndrome virus in Korean domesticated pigs. Vector Borne Zoonotic Dis. 2018;18(8):450-2.

39. Kim KH, Ko MK, Kim N, Kim HH, Yi J. Seroprevalence of severe fever with thrombocytopenia syndrome in southeastern Korea, 2015. J Korean Med Sci. 2017:32(1):29-32.

40. Han MA, Kim CM, Kim DM, Yun NR, Park SW, Han MG, et al. Seroprevalence of severe fever with thrombocytopenia syndrome virus antibodies in rural areas, South Korea. Emerg Infect Dis. 2018;24(5):872-4.

41. Kim UJ, Oh TH, Kim B, Kim SE, Kang SJ, Park KH, et al. Hyperferritinemia as a diagnostic marker for severe fever with thrombocytopenia syndrome. Dis Markers. 2017:2017:6727184. 